\title{
Dipolar-random-field Ising model: An application to garnet films
}

\author{
Alessandro Magni* \\ IEN Galileo Ferraris, INFM, Corso M. d'Azeglio 42, 1-10125 Torino, Italy
}

Gabor Vertesy

Research Institute for Technical Physics and Materials Science, H-1525 Budapest, P.O. Box 49, Hungary

(Received 8 March 1999; revised manuscript received 10 May 1999)

The dipolar-random-field Ising model (DRFIM) recently introduced [A. Magni, Phys. Rev. B 59, 985 (1999)] displays a behavior that can be connected to the magnetization of bidimensional magnetic media. Epitaxial magnetic garnet films seem to be the ideal test material for such a model. In this work the results of the measurements performed on garnet samples are presented, as well as the comparisons with simulation results obtained by the DRFIM. The results prove that a variety of hysteresis loops are well described by the DRFIM. This capability does not derive from the fine tuning of a great number of parameters, but by the interplay of exchange and dipolar interactions.

\section{INTRODUCTION}

The dipolar-random-field Ising model ${ }^{1-3}$ (DRFIM) proved itself apt to describe many different magnetic behaviors, at the level of both the hysteresis loops and the domain structure. We present now a possible application to the description of the magnetization phenomenon in magnetic garnet films.

In the DRFIM, the Hamiltonian is defined on a twodimensional lattice. Two types of spin-spin interaction are present: the spins interact at a next-neighbor level (exchange interaction), and with long-range dipolar interaction. The exchange interaction is ferromagnetic, while the dipolar interaction is antiferromagnetic, thus describing the effect of long-range demagnetizing forces. Last, the interaction of each spin with a frozen disorder is present in the Hamiltonian; this term forces the spin in a preferred direction, chosen at random from the start. The system state is then studied in its evolution under the action of an applied field. Among the results reported in Ref. 1, a regime has been observed in which the domain structure is in the form of stripe domains, while the hysteresis loops present an important nucleation jump, followed by a nearly zero-loss region in which just the motion of the domain walls (DW's) is present. A comparison is then immediate with thin materials such as garnet films, which exhibit similar phenomena. ${ }^{4}$

\section{MODEL DESCRIPTION}

The description of the DRFIM must start from its Hamiltonian, ${ }^{1}$ based on a bidimensional lattice, with periodic boundary conditions.

$$
\aleph=-\frac{J}{2} \sum_{\langle i j\rangle} s_{i} s_{j}+\frac{P}{2} \sum_{\substack{i, j \\ i \neq j}} \frac{s_{i} s_{j}}{r_{i j}^{3}}-V \sum_{i} h_{i}^{c} s_{i}-H \sum_{i} s_{i},
$$

where the four terms are, in sequence: the exchange interaction; the dipolar interaction; the random field; and the external field interaction.

The $J$ and $P$ parameters express the strength of the exchange and dipolar interactions, while the $V$ constant repre- sents the strength of the local random fields $h_{i}^{c}$. The random fields are obtained from a Gaussian distribution with zero mean and unit variance. The exchange interaction acts at a nearest-neighbor level only, while the dipolar interaction is long range, with $r_{i j}=\sqrt{\left(x_{i}-x_{j}\right)^{2}+\left(y_{i}-y_{j}\right)^{2}}$ indicating the relative distance in lattice units. The applied field $H$ acts as the external driving force, and is used to magnetize the system.

Two limit cases can be recovered from this model. When the $P$ parameter is zero, we obtain the random-field Ising model (RFIM) Hamiltonian. ${ }^{5-9}$ When instead the $V$ parameter is zero, we obtain the dipolar Ising Hamiltonian, recently introduced $^{10-13}$ to describe the magnetostatic energy of a system.

The dimensional counterpart of Eq. (1) can be obtained if one knows the correspondence between the $\{J, P, V\}$ constants of the model and the physical phenomena present in a thin magnetic medium that the model simulates. To recover the exchange parameter value, we make the assumption that the exchange interaction between two cells of side $a$, thickness $h$, and opposite magnetic moments $\pm m$ be equal to the energy of the DW's separating the two cells: $J m^{2}=\sigma_{\mathrm{DW}} a h$, where $\sigma_{\mathrm{DW}}$ is the DW energy density. Under the approximation in which the long-range interaction between two cells is dipolar, we recover the dipolar interaction parameter as $P=\mu_{0} / 4 \pi$. Last, the disorder term adds together the effect of different physical phenomena, such as dislocations in the lattice, or the presence of impurities. Therefore, $V$ remains in the Hamiltonian as a free parameter, with the dimensions of a field. The dimensional Hamiltonian can then be written as

$$
\begin{aligned}
\aleph= & -\frac{1}{2} \sigma_{\mathrm{DW}} a h \sum_{\langle i, j\rangle} s_{i} s_{j}+\frac{1}{2} \frac{\mu_{0}}{4 \pi} \frac{m^{2}}{a^{3}} \sum_{\substack{i, j \\
i \neq j}} \frac{s_{i} s_{j}}{r_{i j}^{3}} \\
& -\mu_{0} \operatorname{Vm} \sum_{i} h_{i}^{c} s_{i}-\mu_{0} H m \sum_{i} s_{i}+\frac{I_{s}^{2} U}{2 \mu_{0}},
\end{aligned}
$$

where the spins $s_{i}$ assume the values \pm 1 , and the distances $r_{i j}$ are in lattice units. The magnetic moment of a single cell 
is $m=a^{2} h I_{s} / \mu_{0}$, and $I_{s}$ is the saturation polarization. The last term (zero term $E_{0}$ ) is added to correctly set the zero value of the magnetostatic energy. In fact, if all the cells are separated to an infinite distance from each other, the total magnetostatic energy will be equal to the number of cells times the magnetostatic energy of a single cell $E_{\infty}^{\mathrm{ms}}=\left(\mu_{0} / 2\right) a^{2} h\left(I_{s}^{2} / \mu_{0}^{2}\right) \cdot N$, and $U=N a^{2} h$ is the total system volume. It should be observed that the addition of the correction term in no way changes the dynamic behavior of the system, being just an offset in the energy, nondependent on the system state.

The Hamiltonian is dependent on the spin configuration (system state) $\left\{s_{i}\right\}$ and on the external field $H$. Then, the variation of the Hamiltonian can be written as

$$
\begin{aligned}
d \aleph= & -\sum_{i}\left(\sigma_{\mathrm{DW}} a h \sum_{\langle j\rangle} s_{j}-\frac{\mu_{0}}{4 \pi} \frac{m^{2}}{a^{3}} \sum_{\substack{j \neq i \\
j \neq i}} \frac{s_{j}}{r_{i j}^{3}}+\mu_{0} V m h_{i}^{c}\right. \\
& \left.+\mu_{0} H m\right) d s_{i}-\mu_{0} m\left(\sum_{i} s_{i}\right) d H \\
= & -\mu_{0} m \sum_{i} h_{i} d s_{i}-\mu_{0} m\left(\sum_{i} s_{i}\right) d H,
\end{aligned}
$$

where we have defined the local field $h_{i}$ experienced by $s_{i}$ as

$$
h_{i}=\frac{\sigma_{\mathrm{DW}} a h}{\mu_{0} m} \sum_{\langle j\rangle} s_{j}-\frac{1}{4 \pi} \frac{m}{a^{3}} \sum_{\substack{j \\ j \neq i}} \frac{s_{j}}{r_{i j}^{3}}+V h_{i}^{c}+H .
$$

Equation (3) shows that the system energy can change as a consequence of two possible factors: the inversion of a spin, or a change in the external field.

The stability condition requires that the system state is considered stable when each spin is directed according to the sign of the local field $h_{i}: s_{i}=\operatorname{sgn}\left(h_{i}\right)$. The study we will present is at zero temperature: no temperature fluctuations are considered that can invert a spin in the opposite direction, as long as the applied field $H$ does not change, thus changing the $h_{i}$ value. Moreover, we will assume that the evolution of the system occurs at timescales much shorter than the external field rate of change: a behavior known as rateindependent hysteresis. The consequence of this assumption is that, during an irreversible state change (avalanche) the external field can be considered constant.

\section{CONTROL RATIOS}

In Ref. 1 it was shown that the hysteresis loop properties in the framework of this model depended on just two control ratios: the dipolar to exchange strengths ratio $\mathbf{D}=P / J$, and the disorder to exchange strength ratio $\mathbf{v}=V / J$ [see Eq. (1)]. When the dipolar interaction strength is lower than the exchange coupling $(\mathbf{D}<0.5)$, the hysteresis loop shows two big avalanches, spanning a great part of the lattice. If $\mathbf{D}=0$, just the two saturated system states are possible. Instead, an increase in the value of $\mathbf{D}$ let us observe a smaller nucleation jump. In garnet films, ${ }^{14,15}$ as well as in other materials such as monocrystalline ferrite plates ${ }^{16}$ the hysteresis loops behave in exactly the same way, with a nucleation jump followed by a region characterized by smaller losses,
TABLE I. Model properties under varying control ratios.

\begin{tabular}{lll}
\hline \hline Control ratios & Hysteretic behavior & Domain structure \\
\hline $\mathbf{D}<0.5 ; \mathbf{v}=0$ & Ising like & Saturated state \\
$\mathbf{D}<0.5 ; \mathbf{v}>1$ & RFIM & Cluster domains \\
$0.5<\mathbf{D}<2 ; \mathbf{v}<1$ & garnetlike & Stripe domains \\
\hline \hline
\end{tabular}

typical of DW motion. As the $\mathbf{v}$ ratio is increased, the nucleation jump magnitude decreases to zero. In fact, a high enough value of the disorder $\mathbf{v}$ causes the spins to flip independently, and, as a consequence, the height of the irreversible jumps decreases. The nucleation jump originates by the exchange interaction. A complete magnetization reversal in a field interval $\Delta H \rightarrow 0$ is contrasted by the two other terms in the Hamiltonian: the dipolar interaction and the disorder term. In the region $0.5<\mathbf{D}<2$, where the nucleation jump is still well defined, the dipolar field contribution is strong enough that a disorder to exchange ratio $\mathbf{v} \approx 10^{-2}$ is sufficient to destroy the nucleation jump. As the model approaches the RFIM description instead $(\mathbf{D}<0.5),{ }^{6}$ the dipolar term becoming negligible, a greater $\mathbf{v}$ value is necessary to hinder the infinite avalanche. The DRFIM is able to produce a wide variety of magnetic behaviors, when its two main ratios are tuned. A partial summary is given in Table I.

In our case, three physical parameters appear in the Hamiltonian Eq. (2) known in advance: the saturation polarization $I_{s}$, the DW surface density $\sigma_{\mathrm{DW}}$, and the sample thickness $h$. Two additional parameters are present, that have to be tuned in order to obtain a comparison with real material loops: the disorder strength $V$ and the lattice spacing $a$. The number of spins used in the simulation is important because it allows us to observe the details of the avalanches, but it has been already observed that - beyond a given limit - the loop shape does not change when increasing $N$. Knowing the value of the physical parameters and the value of $V, a$ we obtain

$$
\mathbf{D} \frac{I_{s}^{2} h}{4 \pi \mu_{0} \sigma_{\mathrm{DW}}} ; \quad \mathbf{v}=\left(\frac{I_{s}}{\sigma_{\mathrm{DW}}}\right) a V .
$$

While it appears evident that there is a proportionality between the product $a V$ and the disorder field in the $\mathbf{v}$ ratio, the D ratio is dependent just on the physical parameters of the sample: saturation, thickness, and the DW energy density, and it is therefore known from the start. An interesting property is the $\mathbf{D} \propto h$ dependence: it follows that in this model we will have a value of the $\mathbf{D}$ ratio decreasing to zero with decreasing thickness. Therefore, we can expect that the stripe domain structure, that is a signature of a high dipolar contribution, will seldom be found on very thin films, as in fact it is observed.

\section{OBSERVATIONS AND COMPARISON}

The measured hysteresis loops are obtained in the Faraday effect. The light produced by a halogen lamp is polarized, then traverses the sample perpendicularly. The sample is magnetized with a coil able to produce a field perpendicular to the sample, with $H_{\max }^{\perp} \sim 310^{4} \mathrm{~A} / \mathrm{m}$. The light beam then goes through, in sequence, the objective, a second polarizer, 
TABLE II. Physical parameters of the samples. $I_{s}$, is the saturation polarization; $l$ is the characteristic length; $P_{0}$ is the zero-field stripe domain period: $h$ is the film thickness: $\sigma_{\mathrm{DW}}$ is the DW energy density. Also shown the best fit parameters used $V$, $a$, and the resulting control ratios $\mathbf{D}, \mathbf{v}$.

\begin{tabular}{cccccccccc}
\hline \hline & & \multicolumn{3}{c}{ Physical parameters } & & \multicolumn{2}{c}{ Fit parameters } & \multicolumn{2}{c}{ Control ratios } \\
Sample & $I_{s}(m T)$ & $l(\mu \mathrm{m})$ & $P_{0}(\mu \mathrm{m})$ & $h(\mu \mathrm{m})$ & $\sigma_{\mathrm{DW}}\left(\mathrm{mJ}^{2} \mathrm{~m}^{2}\right)$ & $V(\mathrm{~A} / \mathrm{m})$ & $a(\mu \mathrm{m})$ & $\mathbf{D}$ & $\mathbf{v}$ \\
\hline \multirow{2}{*}{$A$} & 5.18 & 7.90 & 165. & 12.6 & 0.17 & $10^{-10}$ & 80 & 0.126 & $2.0 \times 10^{-13}$ \\
$B$ & 20.5 & 0.58 & 10.5 & 5.4 & 0.19 & $10^{-15}$ & 4 & 0.756 & $8.6 \times 10^{-19}$ \\
$C$ & 16.3 & 3.66 & 56. & 11.8 & 0.77 & $3.10^{-11}$ & 21 & 0.258 & $2.4 \times 10^{-14}$ \\
\hline \hline
\end{tabular}

the ocular, and a photodiode. The signal reported by the photodiode is proportional to the light intensity, which is linked to the sample magnetization. This is due to the fact that, although the Faraday rotation depends upon the wavelength of the light, in white light one can nevertheless correlate changes in magnetization with light variations. This has been carefully tested by measuring the loops in the same material, with white light and He-Ne laser light, and comparing the results with a measurement performed in a PAR vibrating sample magnetometer. No differences have been found.

As shown for example in Ref. 17, a relationship exists between light intensity $L$ and the corresponding sample relative magnetization $I / I_{s}$, depending upon two factors: the angle $(\pi / 2-\varphi)$ between analyzer and polarizer, and the total Faraday rotation of the sample $\theta$. Being often difficult to know with great precision the $\varphi, \theta$ values, it is possible to derive a relationship $I / I_{s}=f\left(L ; L_{+}, L_{-}, L_{0}\right)$, where $L_{+}, L_{-}$ and $L_{0}$ are the light intensities at positive, negative saturation, and remanence, respectively. In our optical bench this relationship is automatically applied, the result being the loop $\{H(A / m), I(T)\}$, where $I$ is obtained by $I / I_{s}$ and the knowledge of $I_{s}$ by vibrating sample magnetometer measurements.

Three garnet samples have been studied, whose physical parameters are described in Table II. The chemical composition of the samples is $(\mathrm{YSmCa})_{3}(\mathrm{FeGe})_{5} \mathrm{O}_{12}$ (samples $A$ and $B)$ and $(\mathrm{YCa})_{3}(\mathrm{FeGeCo})_{5} \mathrm{O}_{12}$ (sample $\left.C\right)$. The samples were chosen to show a variety of loop properties (Figs. 1, 2,3). Although samples $A$ and $B$ share the same chemical composition they behave very differently due to differences in the growth parameters used. Sample $B$ possesses the most typical garnet loop, with a great nucleation step appearing at a fixed field value $H \approx 6000 \mathrm{~A} / \mathrm{m}$, after which the magnetization proceeds exclusively by DW motion, with very low

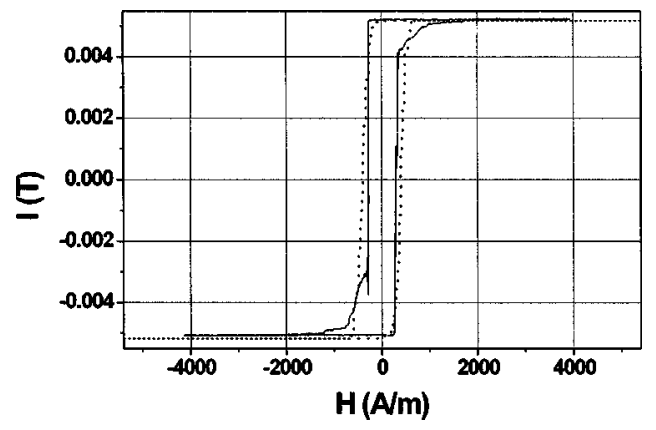

FIG. 1. Sample $A$ hysteresis loop (solid line: experimental; dotted line: simulation). losses. Sample $A$ is more anomalous. The most striking property is the vanishing of the nucleation jump, substituted by a less discontinuous curve. Sample $C$ 's loop is similar to $A$, but the coercive field is much higher.

One of the more relevant physical phenomena present in the magnetization dynamics of garnet films is domain nucleation. This process very often can be observed on the hysteresis loop as a sharp magnetization jump, followed by a successive region in which just DW motion is present. It is not our aim to develop here a detailed discussion on the nucleation phenomenon, but just to observe some similar points, common to both garnet films and our model. Optical investigation shows that during the nucleation process all the domain structure is generated, in the form of elongated stripe domains. ${ }^{18}$ Just in the successive phase of DW motion the stripes change their width under the action of the external field. Likewise, in DRFIM the first reversed spins are forced, by the concurrent action of dipolar and exchange interactions, to generate elongated stripes spanning the whole lattice. Moreover, the collective behavior of the stripes in both the cases of DRFIM and garnet films is similar: the stripes have a self-avoiding tendency, due to the dipolar interactions. So we often observed the presence of stripes elongating in a given direction during an avalanche, then, coming in the proximity of another stripe, suddenly changing direction, to not intersect it. This behavior is the origin of the generation of the maze pattern. The nucleation phase can be observed clearly on the hysteresis loop-as long as the disorder parameter $V$ is not too high-in the form of a sudden, almost vertical, magnetization jump. The field at which nucleation occurs has been calculated ${ }^{1}$ in the limit of low disorder $V \rightarrow 0$ :

$$
H_{n}=4 \frac{\sigma_{\mathrm{DW}}}{I_{s} a}-9 \frac{I_{s} h}{4 \pi \mu_{0} a} .
$$

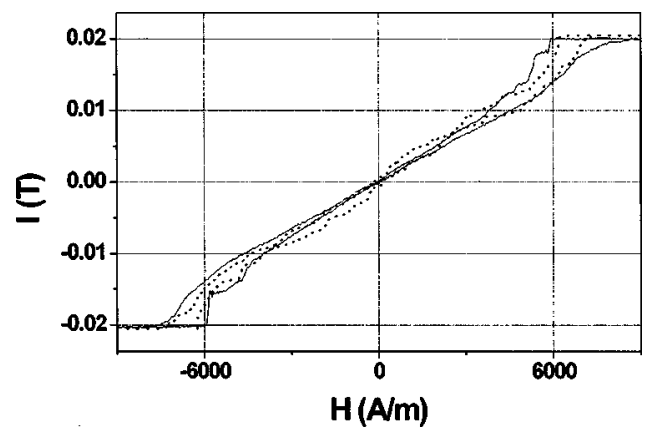

FIG. 2. Sample $B$ hysteresis loop (solid line: experimental; dotted line: simulation). 


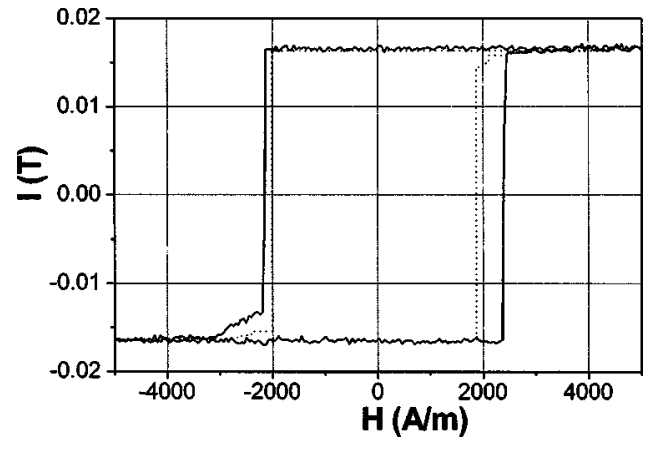

FIG. 3. Sample $C$ hysteresis loop (solid line: experimental; dotted line: simulation).

This calculation has been confirmed by the simulations in the $V \rightarrow 0$ limit.

The most interesting result is the possibility to obtain a good accord of the simulations with the experimental hysteresis loops. The model can describe materials having the typical garnet behavior (sample $B$ ), with a nucleation jump followed by an almost zero-loss region. At the same time, even material loops having squareness $I_{r} / I_{s} \approx l$ (samples $A, C$ ) can be described. The better fits to the physical loops were obtained fixing the $a$ parameter to approximately half the zero field stripe period $P_{0}$. The $a \approx P_{0} / 2$ restriction allowed also to obtain a good fit of the nucleation field value. Consequently, in our model just one free parameter remains, the disorder strength $V$. Being unable to link this parameter to some well known constant describing the disorder in the medium, it has to be tuned up to obtain the better results.

In Table II are shown the simulation parameters used, and the resulting $\mathbf{D}, \mathbf{v}$ values, to be used as a reference to the results in Ref. 1. The modeling of samples $A$ and $C$ shows a low $\mathbf{D}$ value, together with a relatively high $\mathbf{v}$ value. As a consequence both loop shapes are very near to the squared Ising loop, with small deviations just after an almost complete magnetization reversal: the dipolar interaction is not completely negligible, and makes it difficult to completely saturate the lattice. The high $\mathbf{D}$ value of sample $B$ is due to the strong dipolar field present, that severely changes the loop shape compared to samples $A, C$. Let us stress the fact that its value is in no way imposed from the outside, but is just a consequence of the $I_{s}, h, \sigma_{\mathrm{DW}}$ values.

\section{CONCLUSIONS}

We presented a natural application of the dipolar-randomfield Ising model to a physical problem: the garnet film magnetization process. We consider it to be a natural application due to the common features present both in garnet films magnetization dynamics and in DRFIM. First of all, what we named the typical garnet hysteresis loop can be obtained in the model, in a given parameter range. Second, one of the most striking features of the garnet loop, namely the nucleation jump, is present in the DRFIM, too. Last, the domain structure obtained in the DRFIM, in the same parameter range where we obtain the garnet loop, is the stripe domain structure. This domain structure is a common feature of garnet films magnetized in a direction perpendicular to the film plane.

The main feature of this model is the low number of free parameters: the cell side $a$ and the disorder parameter $V$. The first parameter $a$ displayed a strong relationship with a wellknown physical parameter, the zero-field stripe domain period $P_{0}$, so that the next logical step will be to investigate further with the tuning of the $V$ parameter only, that appears to be related to the inner disorder. The basic shape of the loop, given by the ratio $\mathbf{D}$, is known right from the start, from the physical parameters $I_{s}, h, \sigma_{\mathrm{DW}}$. Notwithstanding the low number of parameters present, a great loop shapes variety can be obtained. It must be observed (Tables I and II) that in the case of one of our samples $(B)$ the $\mathbf{D}, \mathbf{v}$ parameters are in the range prescribed for garnets, while in the $A$ and $C$ cases the parameters are midway between the Ising and random-field Ising behaviors. We chose the samples according to the possibility to show a variety of different hysteretic behaviors for these materials. It must be stressed that, to our knowledge, any garnet sample sharing the standard hysteretic properties of sample $B$ can be reproduced by our model. Samples $A$ and $C$ are extreme cases, but, nevertheless, the model parameters can be found in these cases too.

\section{ACKNOWLEDGMENT}

The work was partially (G.V.) supported by Hungarian Scientific Research Fund through Project No. T-026153.
*Electronic address: magni@omega.ien.it

${ }^{1}$ A. Magni, Phys. Rev. B 59, 985 (1999).

${ }^{2}$ U. Nowak, U. Rudiger, and G. Guntherodt, Phys. Rev. B 54, 13017 (1996).

${ }^{3}$ U. Nowak, J. Heimel, T. Kleinefeld and D. Weller, Phys. Rev. B 56, 8143 (1997).

${ }^{4}$ G. Bertotti, Hysteresis in Magnetism (Academic, New York, 1998).

${ }^{5}$ Y. Imry and S. Ma, Phys. Rev. Lett. 35, 1399 (1975).

${ }^{6}$ K. Dahmen and J. P. Sethna, Phys. Rev. Lett. 71, 3222 (1993).

${ }^{7}$ O. Perkovic, K. Dahmen, and J. P. Sethna, cond-mat/9807336 (unpublished).

${ }^{8}$ D. P. Belanger and A. P. Young, J. Magn. Magn. Mater. 100, 272 (1991).

${ }^{9}$ J. Villain, Phys. Rev. Lett. 52, 1543 (1984).
${ }^{10}$ L. C. Sampaio, M. P. de Albuquerque, and F. S. de Menezes, Phys. Rev. B 54, 6465 (1996).

${ }^{11}$ A. B. Mac Isaae, J. P. Whitehead, M. C. Robinson, and K. De'Bell, Phys. Rev. B 51, 16033 (1995).

${ }^{12}$ J. H. Toloza, F. A. Tamarit, and S. A. Cannas, Phys. Rev. B 58, 8885 (1998).

${ }^{13}$ G. Szabo and G. Kadar, Phys. Rev. B 58, 5584 (1998).

${ }^{14}$ G. Vertesy, L. Pust, I. Tomas, and J. Paces, J. Phys. D 24, 1482 (1991).

${ }^{15}$ R. Gemperle, L. Murtinova, and J. Kaczer, Czech. J. Phys., Sect. B 37, 64 (1987).

${ }^{16}$ C. Kooy and U. Enz, Philips Res. Rep. 15, 7 (1960).

${ }^{17}$ I. Tomas, P. Siroky, R. Gemperle, and G. Vertesy, J. Magn. Magn. Mater. 58, 347 (1986).

${ }^{18}$ P. Molho, J. L. Porteseil, Y. Souche, J. Gouzerh, and J. C. S. Levy, J. Appl. Phys. 61, 4188 (1987). 